\title{
Magnitude of Tuberculosis Lymphadenitis in Hawassa University Referral Hospital, Southern Ethiopia
}

\author{
Gemechu Ameya Buli ${ }^{1, *}$, Fekade Yerakly Lukas², Negash Wakgari Amenta ${ }^{3}$ \\ ${ }^{1}$ Medical Laboratory Science Department, College of Medicine and Health Sciences, Arba Minch University, Arba Minch, Ethiopia \\ ${ }^{2}$ Department of Pathology, College of Medicine and Health Sciences, Hawassa University, Hawassa, Ethiopia \\ ${ }^{3}$ Shool of Nursing and Midwifery, College of Medicine and Health Sciences, Hawassa University, Hawassa, Ethiopia
}

Email address:

gemechuameya@gmail.com (G. Ameya), fyerakly@yahoo.com (F. Yerakly),negashwakgari@yahoo.com (N. Wakgari)

\section{To cite this article:}

Gemechu Ameya Buli, Fekade Yerakly Lukas, Negash Wakgari Amenta. Magnitude of Tuberculosis Lymphadenitis in Hawassa University Referral Hospital, Southern Ethiopia. American Journal of Health Research. Vol. 3, No. 2, 2015, pp. 116-120.

doi: 10.11648/j.ajhr.20150302.19

\begin{abstract}
Introduction: In most of developed world Tuberculosis is considered as disease of the past. However; the impact of this disease is overwhelming in developing countries. Tuberculosis lymphadenitis is the main cause of lymphadenophathy in SubSaharan Africa. Hence; this study was aimed to assess the magnitude of Tuberculosis lymphadenitis in patient with enlarged lymph node referred to cytology unity of Hawassa University referral hospital. Methods: A five years trend of Tuberculosis lymphadenitis was retrospectively studied from January, 2009 to January, 2015. Data were entered and analysed by SPSS version 16.00. Logistic regression analyses were used to see the association of different variables. Odds ratios and $95 \%$ confidence interval were computed to determine the presence and strength of association. Results: A total of 1,067 lymph nodes were aspirated in a period of five years. Tuberculosis lymphadenitis was found to be 521(48.8\%). Cervical lymph nodes were recorded to be the highest $286(54.89 \%)$ affected site with tuberculosis lymphadenitis. Age group of $11-20$ years $(\mathrm{AOR}=0.30,95 \% \mathrm{CI}$ : 0.11 , $0.79)$ and 21- 30 years $(\mathrm{AOR}=0.37,95 \% \mathrm{CI}: 0.14,0.97)$ were significantly associated with magnitude of tuberculosis lymphadenitis. Similarly, Cervical lymph nodes $(\mathrm{AOR}=0.62,95 \% \mathrm{CI}: 0.41,0.93)$ were found to be statistically associated with tuberculosis lymphadenitis. Conclusions: A significant percentage of enlarged lymph nodes were caused by Tuberculosis lymphadenitis. Age group and cervical lymph nodes were significantly associated with Tuberculosis lymphadenitis. Pathologists should be conscious of tuberculosis cases whenever they encounter enlarged lymph node to initiates immediate treatments.
\end{abstract}

Keywords: Fine Needle Aspiration Cytology, Southern Ethiopia, Tuberculosis Lymphadenitis

\section{Introduction}

In many developed countries, tuberculosis (TB) is considered as a disease of the past but the impact of this disease is overwhelming in developing countries suffering from high burdens of human immunodeficiency virus [1]. Tuberculosis is an infectious disease caused by bacillus Mycobacterium tuberculosis. It typically affect lung but can also affect other sites which known as extra pulmonary tuberculosis. Over $80 \%$ of the reported TB cases occurred globally were in 22 high burdened countries and Ethiopia is among this countries. In Ethiopia according to WHO 2014 global TB report, the estimated prevalence and incidence of TB cases is 211 and 224 per 100,000 populations, respectively [2].

The tuberculous (TB) pathology is diverse and generating different types of lesions particularly, pulmonary and disseminated TB which contains several distinct Mycobacteral species [3]. TB lymphadenitis is the most common type of disseminated $\mathrm{TB}$ and it is increased in parallel with the incidence of mycobacterial infection in the world $[4,5]$.

During initial phase of infection, the tuberculosis bacilli disseminate to extrapulmonary sites via lymphohematogenous spread. This process results in the influx of lymphocytes and macrophages into infected lymph nodes producing granulomatous inflammatory responses and granuloma formation [6]. And it may occur during primary tuberculous infection or direct extension from a contiguous focus or as a result of reactivation of dormant foci and cause different site of lymph node enlargements commonly cervical, submandibular, inguinal and axillary lymph nodes are involved [7]. There is a wide variation of magnitude of TB 
lymphadenitis in different ethinic groups and in various countries [4, 8-10].

Fine needle aspiration cytology (FNAC) plays a vital role due to its cost effectiveness, simplicity, accuracy, completely safe and quick method for diagnosis of lymphadenopathy and it reduces the need for surgical biopsy [8,9]. The aspirates usually have thick and yellowish appearance with cytological finding of epitheloid granulomas with caseous necrosis, necrosis only without epithelioid granulomas, epithelioid granulomas without necrosis, polymorphs with necrosis with or without epithelioid granulomas, clumps of amorphous acellular debris or caseous necrotic materials, lymphocytes and Langhan giant cells $[10,11]$. The aim of this study was to assess magnitude of TB lymphadenitis in patient with enlarged lymph node referred to cytology unity of Hawassa University referral hospital.

\section{Materials and Methods}

\subsection{Study Design}

A five years trend of TB lymphadenitis was retrospectively studied from January, 2009 to January, 2015 in Hawassa University referral hospital pathology laboratory. This referral hospital provides medical service to fifteen million people and practical training to medicine and health science students. All FNAC reports were recorded in both soft copy and carbon copy in this hospital pathology laboratory. These records were used to assess the magnitude of TB lymphadenitis. All patient with lymphadenopathy referred to pathology laboratory in the study period were considered as study participants.

\subsection{Statistical Analysis}

The data was entered and analyzed using SPSS version 20 statistical software package. Descriptive and summary statistics were done. Both bivariate and multivariate logistic regression analysis was used to determine the association of each independent variable with the dependent variable. Odds ratio with their $95 \%$ confidence intervals were computed to identify the presence and strength of association, and statistical significance was declared if $\mathrm{p}<0.05$.

\subsection{Ethical Consideration}

Ethical approval was obtained from research review board of Hawassa University, College of medicine and health sciences. Permission letter was granted from the pathology department after explaining the aim and purpose of the study before using the data. All obtained data were kept strictly confidential by avoiding personal identifiers.

\section{Results}

A total of 1067 lymph nodes were examined by FNAC from January, 2009 to January, 2015. More than half 550 $(51.55 \%)$ of them were males. They age range of 1 to 85 years, with a mean age of 23.6 years $(\mathrm{SD} \pm 14.66)$ were involved in the study.

Table 1. Magnitude of TB lymphadenitis by age and sex in patient attending Hawassa University referral hospital, southern Ethiopia $(n=521)$.

\begin{tabular}{llll}
\hline \multirow{2}{*}{ Age group } & \multicolumn{3}{l}{ TB lymphadenitis } \\
\cline { 2 - 4 } & Male & Female & Total \\
\hline$<10$ & $53(10.17 \%)$ & $37(7.10 \%)$ & $90(17.27 \%)$ \\
$11-20$ & $94(18.04 \%)$ & $84(16.12 \%)$ & $178(34.16 \%)$ \\
$21-30$ & $79(15.16 \%)$ & $82(15.74 \%)$ & $161(30.90 \%)$ \\
$31-40$ & $25(4.80 \%)$ & $28(5.37 \%)$ & $53(10.17 \%)$ \\
$41-50$ & $7(1.34 \%)$ & $17(3.26 \%)$ & $24(4.60 \%)$ \\
$51-60$ & $6(1.15 \%)$ & $3(0.57 \%)$ & $9(1.72 \%)$ \\
$>61$ & $1(0.19 \%)$ & $5(0.96 \%)$ & $6(1.15 \%)$ \\
Total & $265(50.86 \%)$ & $256(49.14 \%)$ & $521(100 \%)$ \\
\hline
\end{tabular}

Among studied patients with lymph node disease attended in Hawassa University referral hospital, 521 (48.8\%) were showed cytological feature of tuberculosis lymphadenitis. As a whole in these TB lymphadenitis positive patients, there was no great difference between two sexes. In five years trend of TB lymphadenitis, the highest magnitude $(61.10 \%)$ was observed in 2010. The least TB lymphadenitis magnitude was recorded in 2011 which was $43.5 \%$ (Figure 1).

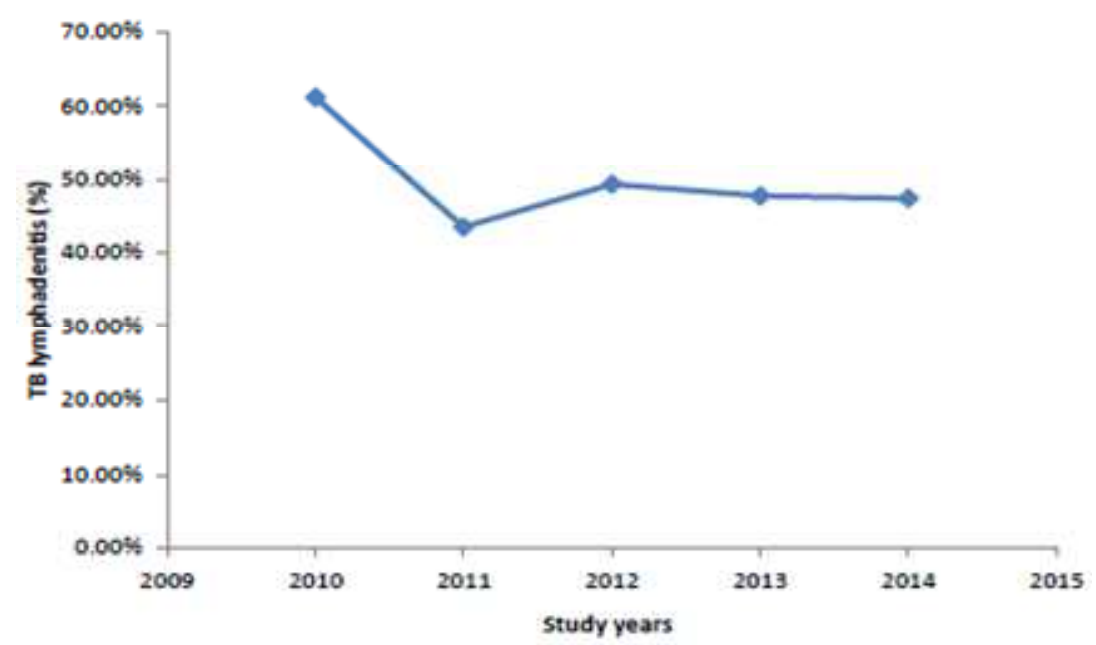

Figure 1. Five years trend of TB lymphadenitis in patient attending Hawassa University referral hospital, southern Ethiopia. 
The highest TB lymphadenitis $178(34.16 \%)$ was observed between 11 to 20 years age whereas the least magnitude 6 $(1.15 \%)$ was recorded in age group above 61 years (Table 1$)$.

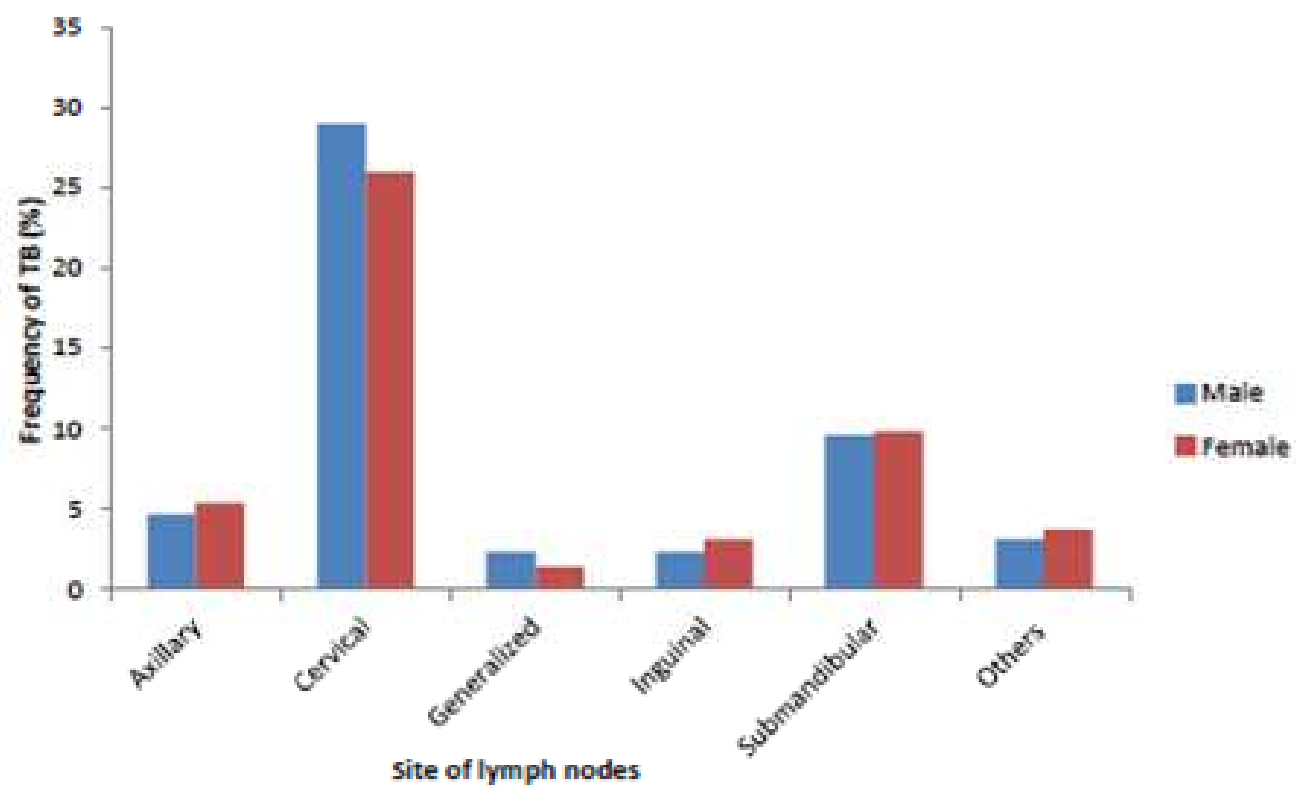

The most affected site was cervical lymph nodes 285 $(54.89 \%)$ whereas the least affected sites were inguinal, submental and supraclavicular (Figure 2).

Figure 2. Tuberculosis lymphadenitis by site of lymph node and sex in patient attending Hawassa University referral hospital, southern Ethiopia.

In multiple logistic regression analysis: age and site of lymph nodes were significantly associated with magnitude Tuberculosis lymphadenitis. Those age group between 11- 20 years were 3.33 times more likely to be diagnosed with TB lymphadenitis than age group of 61 years and above $(\mathrm{AOR}=$
$0.30,95 \%$ CI: $0.11,0.79)$. Similarly age group of $21-30$ years were 2.7 more likely to be diagnosed with Tuberculosis lymphadenitis than age group of 61 years and above $(\mathrm{AOR}=$ $0.37,95 \%$ CI: 0.14, 0.97) (Table 2).

Table 2. Bivariate and Multivariate analyses of factors associated with magnitude of TB lymphadenitis, Hawassa University referral hospital, southern Ethiopia $(n=1067)$.

\begin{tabular}{|c|c|c|c|c|}
\hline \multirow{2}{*}{ Variables } & \multicolumn{2}{|c|}{ Tuberculosis status } & \multicolumn{2}{|l|}{ OR $(95 \%$ CI $)$} \\
\hline & Positive & Negative & Cr COR (95\% CI) & An AOR $(95 \%$ CI $)$ \\
\hline Age in years & 585 & & & $* * *$ \\
\hline$<10$ & 90 & 132 & $0.55(0.21,1.46)$ & $0.60(0.22,1.62)$ \\
\hline $11-20$ & 179 & 129 & $1.000 .27(0.1,0.71)$ & $0.300 .30(0.11,0.79)$ \\
\hline $21-30$ & 161 & 141 & $0000.33(0.12,0.86)$ & ${ }_{* * * *} 0.37(0.14,0.97)$ \\
\hline $31-40$ & 53 & 70 & $1110.49(0.18,1.35)$ & $0000.52(0.19,1.42)$ \\
\hline $41-50$ & 23 & 37 & $0.60(0.21,1.76)$ & $000 \quad 0.66(0.22,1.95)$ \\
\hline$>61$ & 6 & 16 & 111 & 11 \\
\hline \multicolumn{5}{|c|}{ Site of lymph nodes } \\
\hline Inguinal & 51 & 71 & 1 & 1 \\
\hline Cervical & 287 & 232 & $0.58(0.39,0.87)$ & $10.62(0.41,0.93)$ \\
\hline Axillary & 28 & 38 & $00 \quad 0.97(0.53,1.79)$ & $000.94(0.51,1.75)$ \\
\hline Generalized & 19 & 30 & $0001.13(0.76,2.23)$ & $001.06(0.53,2.11)$ \\
\hline Submandibular & 101 & 141 & $1111.01(0.64,1.56)$ & $1.04(0.66,1.64)$ \\
\hline
\end{tabular}

\section{Discussion}

Lymphadenophathy is the most common clinical manifestation of outpatient department and it is very difficult to rule out the cause of disease without cytological examination of enlarged lymph nodes $[12,13]$. In our finding, tuberculosis lymphadenitis found to be $48.8 \%$, which is almost half of enlarged lymph nodes are caused by tuberculosis. This is consistent with study done in
Kathmandu (48.2\%) [14] and Surat, Indian (50.52\%) [15]. The present finding is lower than the studies conducted in northwest part of Ethiopia (69.5\%) [16] and Nepal (66.3\%) [17]; however, it exhibited great difference with study conducted in Houston, Texas (5\%) [18]. The discrepancies between our finding and other studies might be due to differences in socio-demographic characteristics of study participants. There are several factors why this high magnitude has been found in the study area. One of the most 
important possible factors influencing the current high TB magnitude in the study area is poverty, which is closely related to malnutrition, crowded living conditions, lack of access to free or affordable health care services and dependence on traditional healers that can facilitate the transmission of tuberculosis [1]. In addition to this much of the high prevalence of tuberculosis especially in sub-Saharan Africa, has to do with the virulent synergy with HIV [5, 19].

In current study, the rate of TB lymphadenitis was not significantly different between the two sexes. This is in lined with the study done in southwest part of Ethiopia [20]. In our study age group of 11 to 20 and 21 to 30 years had the highest magnitude of tuberculosis lymphadenitis. Similar results were observed in other studies $[16,22]$. This might be due to fact that, the increased exposure of the young adult and adolescent to the environments as they have active social life and also the most affected age group of HIV.

In the present study cervical lymph nodes were significantly associated TB lymphadenitis. This result is supported with several studies carried out in different part of the world [7, 16, 21]. This could be due to the fact that, manifestation of a systemic tuberculosis disease or a unique clinical entity is localized to the neck.

One of the most important factors influencing the current TB epidemic in resource-limited settings like Ethiopia is poverty, which is closely related to malnutrition, crowded living conditions, lack of access to free or affordable health care services, and dependence on traditional healers that can facilitate the transmission of tuberculosis [1]. In Ethiopia, TB diagnosis is also relies on smear microscopy, as it is the most accessible test for TB in the country. TB microscopy services are available at the health centre and hospital levels. Currently all health centers provide AFB (sputum smear) diagnosis, while culture is provided only in few laboratories in the country.

Fine needle aspiration cytology and culture or an excisional biopsy for smear, culture, and pathological examination should be done for effective diagnosis of TB lymphadenitis. AFB smear is often negative in lymph node aspiration specimen due to the low number of organisms, but culture may be positive in up to 80 percent of specimens [23] In addition to this the presence of non caseating granulomas in an individual with risk factors for tuberculosis, especially if the individuals has a positive tuberculin skin test or interferon gamma release assay, supports a clinical diagnosis of tuberculosis lymphadenopathy. However the pathology alone does not differentiate tuberculosis lymphadenopathy from other granulomatous infections.

\section{Conclusion}

A significant percentage of enlarged lymph nodes are caused by tuberculosis lymphadenitis. Age group and cervical lymph nodes were significantly associated with TB lymphadenitis. Therefore; it is an essential to have awareness regarding common presentations of tuberculosis and better to give an attention for the young ages. Pathologists should be conscious of tuberculosis cases whenever they encounter enlarged lymph node to initiates immediate treatments.

\section{Acknowledgement}

We are grateful to Hawassa University, College of medicine and health sciences department of Pathology for permission of data to carry out this study and the staff of pathology laboratory for their cooperation during data collection.

\section{References}

[1] Cegielski JP, McMurray DN. (2004). The relationship between malnutrition and tuberculosis: evidence from studies in humans and experimental animals. Int. J. Tuberc. Lung Dis 8: $286-298$

[2] WHO Library Cataloguing-in-Publication Data. Global tuberculosis report 2014. 1

[3] Mitchison DA. (1985). The action of antituberculosis drugs in short course chemotherapy. Tubercle 66: 219-225.

[4] Corbett EL, Watt CJ, Walker N, Maher D, Williams BG, Raviglione MC, Dye C. (2003). The growing burden of tuberculosis: global trends and interactions with the HIV epidemic. Arch Intern Med 163: 1009-1021.

[5] Aaron L, Saadoun D, Calatroni I, Launay O, Memain N, Vincent $\mathrm{V}$ et al,. (2004). Tuberculosis in HIV infected patients: a comprehensive review. Clin Microbiol Infect 10: 388-398.

[6] Jansy PS, Veronique D, Edmund JDL. (2012). The Role of Transport Mechanisms in Mycobacterium Tuberculosis Drug Resistance and Tolerance. Pharmaceuticals 5: 1210-1235.

[7] Manolidis S, Frenkiel S, Yoskovitch A, Black M. (1993). Mycobacterial infections of the head and neck. Otolaryngol Head Neck Surg 109: 427-433.

[8] Chaturvedi NK, Singh JP, Amita D. (1989). Fine needle aspiration cytology in the diagnosis of tuberculosis lymphadenitis. Indian J Pathol Microbial 32 Suppl 2: 101-4.

[9] Ergete W, Bekele A. (2000). Acid fast bacilli in aspiration smears from tuberculous patients. Ethiopia J Health Dev 14 Suppl 1: 99-104.

[10] Sharma SK, Mohan A. (2004). Extra pulmonary tuberculosis. Indian J. Med. Res.120: 4316-53.

[11] Heerde PV, Miliauskas J, Field A. (2005). Lymph nodes. In: Fine needle aspiration cytology Edited by Orell SR, Sterrett GF, Whitaker D. $4^{\text {th }}$ ed. New York: Churchill Livingstone press $83-124 p$.

[12] Steel BL, Schwartz MR, Ibrahim R. (1995). Fine needle aspiration biopsy in diagnosis of lymphadenopathy in 1,103 patients. Acta Cytologica 39: 76-81.

[13] Ahmad SS, Akhtar S, Akhtar K, Naseem S, Mansoor T. (2005). Study of fine needle aspiration cytology in lymphadenopathy with special reference to Acid-fast staining in cases of tuberculosis. JK Science 7: 1-4. 
[14] Lakhey M, Bhatta CP, Mishra S. (2009). Diagnosis of tubercular lymphadenopathy by fine needle aspiration cytology, acid-fast staining and Mantoux test. J Nepal Med Assoc 48 Suppl 175: 230-33.

[15] Patel MM, Italiya SL, Patel RD, Dudhat RB, Kaptan KR, Baldwa VM. (2013). Role of Fine Needle Aspiration Cytology to Analyze Various Causes of Lymphadenopathy. Natl J Community Med 4 Suppl 3: 489-492.

[16] Muluye D, Biadgo B, W. Gerima E, Ambachew A. (2013). Prevalence of tuberculous lymphadenitis in Gondar University hospital, northwest Ethiopia. BMC Public Health 13: 435.

[17] Shrestha HG. (1989). Extrapulmonary tuberculosis in Histopathological Specimens at T.U. Teaching Hospital. J. of Nepal Medical Association 27: 9-17.

[18] Gonzalez OY, Teeter LD, Thanh BT, Musser JM, Graviss EA. (2003). Extrathoracic tuberculosis lymphadenitis in adult HIV seronegative patients: a population-based analysis in Houston, Texas, USA. Int J Tuberc Lung Dis 7 Suppl 10: 987-93.
[19] Karp CL, Auwaerter PG. (2007). Coinfection with HIV and tropical infectious diseases, Protozoal pathogens. Clin. Infect. Dis 45: 1208-13.

[20] Abebe G, Deribew A, Apers L, Abdissa A, Deribie F, Woldemichael K, et al. (2012). Tuberculosis lymphadenitis in Southwest Ethiopia: a community based cross-sectional study. BMC Public Health 12: 504.

[21] Biadglegne F, Anagaw B, Anagaw B, Tesfaye W, Tessema B. (2013). Tuberculosis lymphadenitis in northwest Ethiopia: implications for public health. Journal of Medical Microbiology 3: 18-24.

[22] Jha BC, Dass A, Nagarkar NM, Gupta R, Singhal S. (2001). Cervical tuberculous lymphadenopathy: changing clinical pattern and concepts in management. Postgrad Med J 77: 185187.

[23] Kasper DL, Braunwald E, fauci AS, Hauser SL, Longo DL, Jemeson JL, LoscalzoJ, (2005). Harrison's principles of internal medicine. $16^{\text {th }}$ Edition, New York: McGraw-Hill Medical publishing Division 